\title{
Prospective evaluation of pentafecta outcomes at 5 years after laparoscopic radical prostatectomy: results of 170 patients at a single center
}

\author{
J. SI-TU*, M. H. LU*, L. Y. LI, Q. P. SUN, X. F. ZHOU, J. G. QIU, X. GAO* \\ Department of Urology, The Third Affiliated Hospital, Sun Yat-sen University, Guangzhou, 510630, China \\ *Correspondence: billurology@126.com \\ ${ }^{*}$ Contributed equally to this work.
}

Received September 14, 2012 / Accepted October 26, 2012

\begin{abstract}
A new and more comprehensive methodology for reporting outcomes after radical prostatectomy (RP) has been proposed: the so-called pentafecta. However, no prior studies reported intermediate- and long-term pentafecta outcomes after laparoscopic RP. We collected prospectively the clinical data of 170 consecutive patients with a minimum 60-month follow-up undergoing laparoscopic RP for clinically localized prostate cancer. International Consultation on Incontinence Questionnaire-Urinary Incontinence Short Form and the Sexual Health Inventory for Men score were used to evaluate the functional outcomes. Logistic regression was used to perform univariable and multivariable analyses. Sixty months after surgery, a pentafecta outcome was achieved by 124 patients $(72.9 \%)$. On univariable regression analysis, patient age at surgery $(P<0.001)$, body mass index $(P=0.031)$, pathological T stage $(P<0.001)$ and prostate volume $(P=0.003)$ were significantly associated with pentafecta rates. On multivariable analysis, only patient age at surgery (odds ratio 0.95 ; $P=0.006$ ) and pathological T stage (odds ratio $0.82 ; P<0.001$ ) were independent predictors of pentafecta rates. Using validated questionnaires to assess functional outcomes, for the first time, we evaluated pentafecta outcomes at 5 years after laparoscopic RP. This approach may be beneficial and could be used when counseling patients with clinically localized prostate cancer.
\end{abstract}

Key words: prostate cancer, laparoscopic radical prostatectomy, urinary continence, potency, pentafecta outcome

Although radical retropubic prostatectomy remains the gold standard for the treatment of localized prostate cancer, laparoscopic radical prostatectomy (LRP) is now an attractive alternative to open prostatectomy, offering significant advantages in terms of a higher percentage of early catheter removal and reduced blood loss $[1,2]$. Historically, the ideal outcome of radical prostatectomy has been measured by achievement of the trifecta rate, which denotes the concurrent attainment of continence and potency with no evidence of biochemical recurrence (BCR) [3]. However, in the prostate-specific antigen

Abbreviations: BCR, biochemical recurrence; BMI, body-mass index; CI, confidence interval; ICIQ-UI SF, International Consultation on Incontinence Questionnaire-Urinary Incontinence Short Form; IQR, interquartile range; LRP, laparoscopic radical prostatectomy; OR, odds ratio; PDE5, phosphodiesterase type 5; PSA, prostate-specific antigen; PSM, positive surgical margins; SD, standard deviation; SHIM, Sexual Health Inventory for Men.
(PSA) era, prostate cancer is frequently diagnosed in younger and healthier patients who have higher expectations from the advanced minimally invasive surgical technologies [4]. Mere trifecta is no longer an ideal outcome measure to meet the demands of such patients [5].

Recently, Patel et al. proposed a new and more comprehensive methodology for reporting outcomes after radical prostatectomy: the so-called pentafecta. In the pentafecta rate, complications and surgical margin status, along with the three major outcomes classically reported in trifecta rates (continence, potency, and BCR-free survival rates) were included [6]. However, to our knowledge, no prior study has prospectively evaluated pentafecta outcomes at 5 years after LRP.

The purpose of the present study is to evaluate the pentafecta outcome and its preoperative predictors in a series of consecutive patients who underwent bilateral nerve-sparing LRP. 


\section{Patients and methods}

Patients. From June 2002 to June 2011, 712 consecutive patients underwent LRP by a single experienced surgeon (X.G.). Following institutional review board approval, the data were prospectively collected into a database. Informed consent was obtained from each patient prior to enrollment in the study. For the present study, we extracted all 434 consecutive cases that had a minimum follow-up of 60 months. Once we excluded those patients who underwent non-nerve sparing LRP, and those who did not fill in the International Consultation on Incontinence Questionnaire-Urinary Incontinence Short Form (ICIQ-UI SF) or the Sexual Health Inventory for Men (SHIM) score $\leq 21,170$ patients remained for the present analysis.

Surgical procedure. LRP was performed using the Montsouris technique, with some modifications by us [7]. In brief, the bilateral endopelvic fascia of the prostate is dissected longitudinally toward the apex and puboprostatic ligament section. When the bladder neck is transected and its posterior wall opened, the vas deferens and seminal vesicles are dissected carefully to avoid injury to the pelvic plexus. The Denonvilliers' fascia is exposed and opened, and the posterior plane of the prostate is created and extended to the apex of the prostate. The lateral vascular pedicle of the prostate is then dissected using a harmonic scissors. A sharp scissor is used to cut the anterior aspect of the urethra close to the prostate apex. Preservation of the neurovascular bundles was performed using an intra-fascial technique [8].The following criteria were used to define prostate cancer patients in whom pelvic lymph node dissection was performed because of a high likelihood of nodal disease: PSA $\geq 20 \mathrm{ng} / \mathrm{ml}$, biopsy Gleason score $\geq 8$, or clinical suspicion of lymph node metastases [9].

Patients were suggested to use phosphodiesterase type 5 (PDE5) inhibitors at least three times a week until return of sexual function. No patients performed penile rehabilitation with prostaglandins injections or vacuum device.

Hisopathologic analysis. All surgical specimens were inked, and the prostate then sliced in entirety. The apex and bladder-neck cones were sectioned in the sagittal plane. The remaining portion was sectioned transversely at 4-mm intervals. Positive surgical margins (PSMs) were defined as the presence of tumor on the inked surface of the specimen [7]. All specimens were examined by a single pathologist and histopathologic staging was performed according to the 2002 TNM system [10].

Definition of BCR, continence, potency, complications, and pentafecta rate. BCR was defined as two consecutive PSA levels of $>0.2 \mathrm{ng} / \mathrm{ml}$ after LRP [11]. Postoperative follow-up comprised a clinical exam and PSA levels at 3, 6, 9, and $12 \mathrm{mo}$; and then at every 6 mo subsequently. In these cases of BCR, salvage therapy was administered based on the decision of the attending urologist.

Urinary continence and potency were evaluated using the ICIQ-UI SF and the SHIM questionnaires, respectively. Patients completed the questionnaires before and 60 months after LRP. All of the patients reporting 'no leak' for the question 'How often do you leak urine?' on the ICIQ-UI SF were defined as continent [12]. Those patients with a SHIM score $\geq 22$ were considered as potent, regardless of the use of PDE5 inhibitors [13].

Complications occurring during the surgical procedure or within 3 mo after LRP were recorded and classified according to the modified Clavien grading system [14]. Patient comorbidity was evaluated using the Charlson comorbidity index score [15].

Outcomes included in the evaluation of the pentafecta rate were complications and surgical margin status combined with the three outcomes classically reported in the trifecta (continence, potency, and BCR-free survival rates). Only those patients who met all criteria were considered to reach the pentafecta. Success in each of the parameters was defined as: absence of perioperative complications (grade 0 on Clavien grading system), negative surgical margins, and achievement of trifecta outcomes (continent, potent, and BCR-free survival).

Statistical analysis. Continuous parametric variables were reported as the mean value \pm standard deviation (SD) or as the median values and interquartile range (IQR). Mann-Whitney $U$-test and chi-square tests were used to compare continuous and categorical variables as appropriate. A logistic regression model was used to perform univariable and multivariable analyses. A two-tailed test with $P<0.05$ was considered statistically significant. All statistical tests were performed using SPSS v.16.0 (SPSS Inc., Chicago, IL, USA).

\section{Results}

Table 1 summarizes the clinical data on the 170 patients evaluated. In total, lymphadenectomy was performed in 23 of 124 patients achieving pentafecta and 9 of 46 patients not achieving pentafecta $(18.5 \%$ vs $19.6 \%, P>0.05)$. There was no statistically significant difference in the median number of removed lymph nodes ( 8.7 vs $9.3, P>0.05)$. No positive findings were present in all removed nodes. The median follow-up of the whole cohort was 71 months (range 60-112). Sixty months after LRP, 165 patients (97.1\%) were continent and 128 (75.3\%) were potent. Eighteen patients $(10.6 \%)$ had either salvage hormone therapy ( $n=10,5.9 \%)$ or radiation therapies $(n=8,4.7 \%)$ for PSA relapse. The overall trifecta rate in our study, at $60 \mathrm{mo}$, was $79.4 \%$. The most common reasons for not reaching the trifecta were impotence, followed by BCR. On the whole, a pentafecta outcome was achieved by 124 patients (72.9\%). Those patients who achieved a pentafecta outcome were significantly younger than those who did not (61.5 \pm 5.8 vs $65.2 \pm 6.3$ years, respectively; $P=0.003)$; they also had a lower body-mass index (BMI) (23.4 \pm 3.3 vs $25.2 \pm 3.1$, respectively; $P=0.017)$, a lower pathological T stage $(P=0.001)$ and a lower median prostate volume (35 vs $37 \mathrm{ml}$, respectively; $P=0.007$ ) (Table 1).

Table 2 summarizes the univariable and multivariable analyses for pentafecta rate. On univariable regression analy- 
Table 1. Association of pentafecta outcome with clinicopathologic characteristics of the 170 patients

\begin{tabular}{|c|c|c|c|}
\hline Characteristics & Pentafecta outcome achieved $(n=124)$ & Pentafecta outcome not achieved $(n=46)$ & $P$ value \\
\hline Age (years, mean $\pm S D$ ) & $61.5 \pm 5.8$ & $65.2 \pm 6.3$ & 0.003 \\
\hline $\mathrm{BMI}(\operatorname{mean} \pm \mathrm{SD})$ & $23.4 \pm 3.3$ & $25.2 \pm 3.1$ & 0.017 \\
\hline Charlson comorbidity index (median and IQR) & $2(1-2)$ & $2(1-2)$ & 0.72 \\
\hline Baseline SHIM score (median and IQR) & $24(23-25)$ & $24(23-25)$ & 0.56 \\
\hline PSA (ng/mL) (median and IQR) & 7.9(4.9-10.6) & $8.2(5.5-11.2)$ & 0.29 \\
\hline Biopsy Gleason score & & & 0.73 \\
\hline$\leq 6$ & $70(56.5 \%)$ & $27(58.7 \%)$ & \\
\hline 7 & $38(30.6 \%)$ & $14(30.4 \%)$ & \\
\hline $8-10$ & $16(12.9 \%)$ & $5(10.9 \%)$ & \\
\hline Clinical T stage & & & 0.09 \\
\hline $\mathrm{cT} 1$ & $68(54.8 \%)$ & $21(45.7 \%)$ & \\
\hline $\mathrm{cT} 2$ & $56(45.2 \%)$ & $25(54.3 \%)$ & \\
\hline Prostate volume (specimen) (ml, median and IQR) & $35(27-43)$ & $37(29-46)$ & 0.007 \\
\hline Pathological T stage & & & 0.001 \\
\hline pT2a-c & $74(59.7 \%)$ & $18(39.1 \%)$ & \\
\hline pT3-4 & $50(40.3 \%)$ & $28(60.9 \%)$ & \\
\hline Specimen Gleason score & & & 0.77 \\
\hline$\leq 6$ & $35(28.2 \%)$ & $11(23.9 \%)$ & \\
\hline 7 & $63(50.8 \%)$ & $13(28.3 \%)$ & \\
\hline 8-10 & $26(21.0 \%)$ & $22(47.8 \%)$ & \\
\hline Complications & $7(5.6 \%)$ & $2(4.3 \%)$ & 0.81 \\
\hline Surgical margin status & & & $<0.001$ \\
\hline Positive & $0(0)$ & $9(19.6 \%)$ & \\
\hline Negative & $124(100 \%)$ & $37(80.4 \%)$ & \\
\hline
\end{tabular}

Abbreviations: SD, standard deviation; BMI, body-mass index; IQR, interquartile range;SHIM, Sexual Health Inventory for Men; PSA, prostate-specific antigen.

Table 2. Univariable and multivariable analyses for pentafecta outcome

\begin{tabular}{|c|c|c|c|c|}
\hline \multirow{2}{*}{ Variable } & \multicolumn{2}{|c|}{ Univariable analysis } & \multicolumn{2}{|c|}{ Multivariable analysis } \\
\hline & OR $(95 \% \mathrm{CI})$ & $P$ value & OR $(95 \% \mathrm{CI})$ & $P$ value \\
\hline Age & $0.97(0.89-0.99)$ & $<0.001$ & $0.95(0.82-0.98)$ & 0.006 \\
\hline BMI & $0.94(0.91-0.98)$ & 0.031 & $0.92(0.88-1.02)$ & 0.63 \\
\hline Charlson comorbidity index & $1.01(0.97-1.05)$ & 0.64 & $0.98(0.89-1.02)$ & 0.75 \\
\hline Baseline SHIM score & $1.17(0.84-1.51)$ & 0.28 & $0.89(0.73-1.06)$ & 0.49 \\
\hline PSA level (ng/ml) & $1.14(1.01-1.38)$ & 0.056 & $0.86(0.64-1.23)$ & 0.17 \\
\hline Prostate volume (ml) & $0.88(0.79-1.04)$ & 0.003 & $0.96(0.87-1.01)$ & 0.41 \\
\hline Biopsy Gleason score & $1.07(0.53-1.97)$ & 0.053 & $1.26(1.13-1.79)$ & 0.34 \\
\hline Clinical T stage & $0.96(0.84-1.13)$ & 0.08 & $1.02(0.92-1.53)$ & 0.28 \\
\hline Pathological T stage & $0.76(0.65-0.98)$ & $<0.001$ & $0.82(0.69-0.93)$ & $<0.001$ \\
\hline Specimen Gleason score & $1.02(0.54-1.97)$ & 0.51 & $0.72(0.34-1.45)$ & 0.96 \\
\hline
\end{tabular}

Abbreviations: OR, odds ratio; CI, confidence interval; BMI, body-mass index; SHIM, Sexual Health Inventory for Men; PSA, prostate-specific antigen.

sis, patient age at surgery (odds ratio [OR]: 0.97; $P<0.001)$, BMI (OR:0.94; $P=0.031$ ), pathological T stage (OR:0.76; $P<0.001$ ), and prostate volume (OR:0.88; $P=0.003$ ) were significantly associated with pentafecta rates. On multivariable analysis, only patient age at surgery (OR: 0.95; $P=0.006)$ and pathological T stage (OR: $0.82 ; P<0.001)$ were independent predictors of pentafecta rates 60 months after LRP (Table 2).

\section{Discussion}

In the present study, based on the use of validated questionnaires to assess functional outcome, we found that $72.9 \%$ of our patients undergoing nerve-sparing LRP reached the pentafecta outcome 60 months after surgery. Moreover, patients' age at surgery and pathological $\mathrm{T}$ stage were independent predictors of pentafecta rates on multivariable analysis. 
Although the primary goal of radical prostatectomy is the complete extirpation of the primary tumor, patients' satisfaction could be negatively affected by the presence of urinary incontinence, impotency, or both in the postoperative period. Thus, there is a need to include both oncological and functional outcomes in the assessment of success after radical prostatectomy, as was initially reported by Salomon et al. in 2003 [16]. Subsequently, Bianco et al. [17] coined the term 'trifecta outcome' to identify patients who were continent, potent, and free of PSA progression after surgery. Specifically, the authors reported that $60 \%$ of 758 patients undergoing radical retropubic prostatectomy achieved trifecta outcomes $2 \mathrm{yr}$ after surgery. In an updated publication from the same group, a trifecta rate of $62 \%$ at $4 \mathrm{yr}$ after surgery was reported [3]. Similarly, Shikanov et al. [18] showed overall trifecta rates of $71 \%$ at 12 mo after surgery when applying subjective continence and potency definitions. Obviously, the trifecta rate after such a short postoperative follow-up clearly does not reflect actual cancer control, as the risk of BCR clearly persists even beyond $5 \mathrm{yr}$ after radical prostatectomy and hence does not represent patient satisfaction [19].

Another shortcoming of trifecta outcomes regards postoperative complications. Patients who experience surgical complications, which can potentially affect postoperative satisfaction (e.g. rectal injury), may still achieve trifecta. Recently, Novara et al. [20] reported their trifecta rates of $57 \%$ in 242 consecutive robot-assisted LRPs with a minimum 12-mo follow-up. However, they reported postoperative complication rates as high as $21.6 \%$ in a similar cohort of patients. Specially, 3\% (12 of 405) of the patients had a major complication (grade III and IV on Clavien grading), including 11 patients who required reoperation [21]. Thus, although encouraging trifecta rates were reported, these results may not reflect true patient-satisfaction rates.

Keeping the limitations of trifecta in mind, Patel et al. [6] recently proposed a more comprehensive method of outcomes analysis following radical prostatectomy, called the 'pentafecta' , which adds perioperative complications and PSM rates to the major outcomes currently reported as the trifecta rates. They evaluated 332 consecutive patients who were preoperatively potent, continent, underwent bilateral nerve sparing robotassisted LRP and showed $70.8 \%$ achieved the pentafecta at 12 months. On multivariable analysis, patient age $(P=0.009)$ was the only factor independently associated with the pentafecta. However, to the best of our knowledge, prospective studies reporting intermediate- and long-term pentafecta outcomes after LRP are still lacking.

The overall trifecta rate in our cohort, at $60 \mathrm{mo}$, was $79.4 \%$. When combining the trifecta outcomes with PSMs and complications, a successful outcome was achieved in $72.9 \%$ of the patients (pentafecta rate). The most common reason for failure to reach the pentafecta in an individual outcome was impotence (24.1\%). We therefore hypothesize that the $6.5 \%$ difference between the trifecta and pentafecta rates in the present study could represent those patients who had a suboptimal outcome and potentially are not fully satisfied with LRP.

In the present study, we found that patient age at surgery, BMI, pathological T stage, and prostate volume were significantly associated with the pentafecta rates on univariable analysis. However, on multivariable analysis, only patient age and pathological $\mathrm{T}$ stage were independent predictors of pentafecta rates. Our results are not surprising because pathologic stage is regarded as one the most important predictors for PSMs and BCR after radical prostatectomy. Menon et al. [22] recently analyzed long-term oncologic outcomes in a series of 1384 consecutive LRPs and found, on multivariable analysis, the strongest predictors of BCR were pathologic stage and specimen Gleason grade. With regard to the correlation between patient age and functional outcomes after radical prostatectomy, Mendiola et al. [23] evaluated age-stratified functional outcomes in 338 consecutive LRPs and showed that younger men achieved subjective continence and potency significantly earlier than older men. Similarly, Rogers et al. [24] assessed potency and continence rates with self-administered questionnaires in 369 patients who underwent LRP and found younger men had higher continence and potency rates at $1 \mathrm{yr}$ after LRP when compared with older men, suggesting the clear association between patient age with functional outcome.

The major strengths of our present study include the use of validated questionnaires to evaluate both urinary continence and potency serially, and intermediate and long-term followup after surgery. Furthermore, all LRPs were performed by one single surgeon, using the same surgical technique. On the whole, these results could be useful for patient counseling on the intermediate- and long-term pentafecta outcomes after LRP for prostate cancer. One of the limitations of the study is the number of evaluated patients, which was not particularly large. Consequently, further studies applying the proposed pentafecta algorithm in larger patient populations are needed to validate the current observations. Another relevant limitation is that the pentafecta system is represented by its application only in preoperatively continent and potent patients who receive bilateral nerve-sparing radical prostatectomy. Recently, Ficarra et al. [25] described a novel SCP (Survival, Continence, and Potency classification) system that they proposed could offer the opportunity to appropriately classify all patients who undergo radical prostatectomy according to the oncologic and functional outcomes of relevance to them on an individual basis. However, as far as we know, no validation studies of the novel system, retrospectively or prospectively, to investigate its utility in reporting outcomes in different radical prostatectomy populations are available.

In summary, for the first time, we evaluated prospectively pentafecta outcomes at 5 years after LRP using validated questionnaires to assess functional outcomes. Patient age at surgery and pathological $\mathrm{T}$ stage were independently found to be associated with pentafecta rates. This approach may be beneficial and could be used when counseling patients with clinically localized prostate cancer. 
Acknowledgements: This study was supported by Program of 5010 of Sun-Yat Sen University (2007028 to X.G.), Key Project of Chinese Ministry of Health (to X.G.), National Natural Science Foundation of China (30772178 to X.G. and 81001139 to L.Y.L), the Science and Technology Foundation of the Guangdong Province (2012A030400009 to X.G.), and the Fundamental Research Funds for the Central Universities (11ykpy39 to L.Y.L and to X.G.).

\section{References}

[1] FICARRA V, NOVARA G, ARTIBANI W, CESTARI A, GALFANO A, et al. Retropubic, laparoscopic, and robot-assisted radical prostatectomy: a systematic review and cumulative analysis of comparative studies. Eur Urol 2009; 55: 1037-63. http://dx.doi.org/10.1016/j.eururo.2009.01.036

[2] GUAZZONI G, CESTARI A, NASPRO R, RIVA M, CENTEMERO A, et al. Intra- and peri-operative outcomes comparing radical retropubic and laparoscopic radical prostatectomy: results from a prospective, randomised, single-surgeon study. Eur Urol 2006; 50: 98-104. http://dx.doi.org/10.1016/ j.eururo.2006.02.051

[3 EASTHAM JA, SCARDINO PT, KATTAN MW. Predicting an optimal outcome after radical prostatectomy: the trifecta nomogram. J Urol 2008; 179: 2207-10; discussion 10-1. http:// dx.doi.org/10.1016/j.juro.2008.01.106

[4] GUMULEC J, MASARIK M, KRIZKOVA S, HLAVNA M, BABULA P, et al. Evaluation of alpha-methylacyl-CoA racemase, metallothionein and prostate specific antigen as prostate cancer prognostic markers. Neoplasma 2012; 59: 191-201. http://dx.doi.org/10.4149/neo 2012025

[5] PATEL VR, ABDUL-MUHSIN HM, SCHATLOFF O, COELHO RF, VALERO R, et al. Critical review of ,pentafecta' outcomes after robot-assisted laparoscopic prostatectomy in high-volume centres. BJU Int 2011; 108: 1007-17. http:// dx.doi.org/10.1111/j.1464-410X.2011.10521.x

[6] PATEL VR, SIVARAMAN A, COELHO RF, CHAUHAN S, PALMER KJ, et al. Pentafecta: a new concept for reporting outcomes of robot-assisted laparoscopic radical prostatectomy. Eur Urol 2011; 59: 702-7. http://dx.doi. org/10.1016/j.eururo.2011.01.032

[7] GAO X, JIAN-HUA Z, LI L-Y, QIU J-G, PU X-Y. Laparoscopic radical prostatectomy: oncological and functional results of 126 patients with a minimum 3-year follow-up at a single Chinese institute. Asian Journal of Andrology 2009; 11: 548-56. http://dx.doi.org/10.1038/aja.2009.42

[8] STOLZENBURG JU, SCHWALENBERG T, HORN LC, NEUHAUS J, CONSTANTINIDES C, et al. Anatomical landmarks of radical prostatecomy. Eur Urol 2007; 51: 629-39. http://dx.doi.org/10.1016/j.eururo.2006.11.012

[9] CAGIANNOS I, KARAKIEWICZ P, EASTHAM JA, OHORI $\mathrm{M}, \mathrm{RABBANI} F$, et al. A preoperative nomogram identifying decreased risk of positive pelvic lymph nodes in patients with prostate cancer. J Urol 2003; 170: 1798-803. http://dx.doi. org/10.1097/01.ju.0000091805.98960.13

[10] GREENE FL. The American Joint Committee on Cancer: updating the strategies in cancer staging. Bull Am Coll Surg 2002; 87: 13-5.
[11] MOTTET N, BELLMUNT J, BOLLA M, JONIAU S, MASON $\mathrm{M}$, et al. EAU guidelines on prostate cancer. Part II: Treatment of advanced, relapsing, and castration-resistant prostate cancer. Eur Urol 2011; 59: 572-83. http://dx.doi.org/10.1016/ j.eururo.2011.01.025

[12] AVERY K, DONOVAN J, PETERS TJ, SHAW C, GOTOH M, et al. ICIQ: a brief and robust measure for evaluating the symptoms and impact of urinary incontinence. Neurourol Urodyn 2004; 23: 322-30. http://dx.doi.org/10.1002/nau.20041

[13] CAPPELLERI JC, ROSEN RC. The Sexual Health Inventory for Men (SHIM): a 5-year review of research and clinical experience. Int J Impot Res 2005; 17: 307-19. http://dx.doi. org/10.1038/sj.ijir.3901327

[14] DINDO D, DEMARTINES N, CLAVIEN PA. Classification of surgical complications: a new proposal with evaluation in a cohort of 6336 patients and results of a survey. Ann Surg 2004; 240: 205-13. http://dx.doi.org/10.1097/01. sla.0000133083.54934.ae

[15] CHARLSON M, SZATROWSKI TP, PETERSON J, GOLD J. Validation of a combined comorbidity index. J Clin Epidemiol 1994; 47: 1245-51. http://dx.doi.org/10.1016/08954356(94)90129-5

[16] SALOMON L, SAINT F, ANASTASIADIS AG, SEBE P, CHOPIN D, et al. Combined reporting of cancer control and functional results of radical prostatectomy. Eur Urol 2003; 44: 656-60. http://dx.doi.org/10.1016/j.eururo.2003.09.009

[17] BIANCO FJ, JR., SCARDINO PT, EASTHAM JA. Radical prostatectomy: long-term cancer control and recovery of sexual and urinary function („trifecta“). Urology 2005; 66: 83-94. http://dx.doi.org/10.1016/j.urology.2005.06.116

[18] SHIKANOV SA, ZORN KC, ZAGAJA GP, SHALHAV AL. Trifecta outcomes after robotic-assisted laparoscopic prostatectomy. Urology 2009; 74: 619-23. http://dx.doi. org/10.1016/j.urology.2009.02.082

[19] AMLING CL, BLUTE ML, BERGSTRALH EJ, SEAY TM, SLEZAK J, et al. Long-term hazard of progression after radical prostatectomy for clinically localized prostate cancer: continued risk of biochemical failure after 5 years. J Urol 2000; 164: 101-5. http://dx.doi.org/10.1016/S00225347(05)67457-5

[20] NOVARA G, FICARRA V, D'ELIA C, SECCO S, CAVALLERI $S$, et al. Trifecta outcomes after robot-assisted laparoscopic radical prostatectomy. BJU Int 2011; 107: 100-4. http://dx.doi. org/10.1111/j.1464-410X.2010.09505.X

[21] NOVARA G, FICARRA V, D'ELIA C, SECCO S, CAVALLERI $S$, et al. Prospective evaluation with standardised criteria for postoperative complications after robotic-assisted laparoscopic radical prostatectomy. Eur Urol 2010; 57: 363-70. http://dx.doi.org/10.1016/j.eururo.2009.11.032

[22] MENON M, BHANDARI M, GUPTA N, LANE Z, PEABODY JO, et al. Biochemical recurrence following robot-assisted radical prostatectomy: analysis of 1384 patients with a median 5-year follow-up. Eur Urol 2010; 58: 838-46. http://dx.doi. org/10.1016/j.eururo.2010.09.010

[23] MENDIOLA FP, ZORN KC, MIKHAIL AA, LIN S, ORVIETO MA, et al. Urinary and sexual function outcomes among different age groups after robot-assisted laparoscopic 
prostatectomy. J Endourol 2008; 22: 519-24. http://dx.doi. org/10.1089/end.2006.9845

[24] ROGERS CG, SU LM, LINK RE, SULLIVAN W, WAGNER A, et al. Age stratified functional outcomes after laparoscopic radical prostatectomy. J Urol 2006; 176: 2448-52. http://dx.doi. org/10.1016/j.juro.2006.07.153
[25] FICARRA V, SOORIAKUMARAN P, NOVARA G, SCHATLOFF O, BRIGANTI A, et al. Systematic review of methods for reporting combined outcomes after radical prostatectomy and proposal of a novel system: the survival, continence, and potency (SCP) classification. Eur Urol 2012; 61: 541-8. http://dx.doi.org/10.1016/j.eururo.2011.11.042 